\title{
Test Results Follow-Up: A Systematic Review
}

\author{
Shai Gavi, DO, MPH, Vishal Ranpura, MD, Sanjaykumar Hapani, MD, Abdo Kataya, MD, and \\ Bala Prakash, MD
}

State University of New York at Stony Brook, Stony Brook, NY, USA.

$\mathrm{J}$ Gen Intern Med 28(2):175

DOI: $10.1007 / \mathrm{s} 11606-012-2281-4$

(c) Society of General Internal Medicine 2012

To the Editors:-We applaud the work by Callen et al., ${ }^{1}$ as it highlights a tremendous patient safety issue. As hospitalists, one of our greatest concerns has been the potential neglect in the ambulatory setting of the incidental radiological findings identified in hospitalized patients. The fact that these findings are not the center of attention during the hospital stay makes these findings less likely to be addressed in the medical chart, discussed with the patient, or communicated to the next provider to assure appropriate follow-up.

We evaluated the prevalence of such incidental radiological finding in a cohort of 720 patients that included 371 men and 349 women, with a mean age of $61 \pm 19.8$ years and $63.0 \pm 20.7$ years, respectively. The number of radiological examinations in these patients included $663(92.1 \%)$ x-rays of the chest or abdomen, 330 (45.8\%) computed tomography imaging (CT) scans of chest or abdomen/ pelvis, $142(19.7 \%)$ ultrasounds of abdomen or kidneys, and $19(2.6 \%)$ magnetic resonance imaging (MRI) scans of the abdomen.

An alarming $35.2 \%$ of abdominal or chest CTs revealed incidental radiological findings that required outpatient follow-up. The most common finding was pulmonary nodules, which accounted for $50.4 \%$ of the total. The distribution of the size of the pulmonary nodules was $<5 \mathrm{~mm}$ (45.2\%), 5-9 mm (30.1\%), and > $10 \mathrm{~mm}(24.7 \%)$. Thyroid nodules and adrenal nodules were the second most common

incidental finding, at $14 \%$ each. Less common incidental radiological findings on $\mathrm{CT}$ included aortic aneurysm (12.4\%), liver lesions (7.0\%), pancreatic lesions (2.3\%), and splenic lesions $(2.3 \%)$. The prevalence of incidental findings on ultrasound of the abdomen was lower, affecting $5.6 \%$ of patients (eight patients). Identification of incidental findings in patients undergoing chest and a bdominal x-ray was found to be only $1.9 \%$ (13 patients). Patients with incidental radiological findings were more likely to be older and smokers.

Our findings demonstrate a significant number of incidental radiological findings in hospitalized general medicine patients that require post-discharge follow-up in the ambulatory setting. Given the high number of such incidental findings and that they are not the focus of the hospitalization, a systems approach to the communication of these findings is much needed. ${ }^{2}$

Corresponding Author: Shai Gavi, DO, MPH; State University of New York at Stony Brook, Section of Hospital Medicine, HSC T16, Room 20, Stony Brook, NY 11794, USA (e-mail: shai.gavi@stonybrookmedicine.edu).

\section{REFERENCES}

1. Callen JL, Westbrook JI, Georgiou A, et al. Failure to follow-up test results for ambulatory patients: A systematic review. J Gen Intern Med. 2012;27:1334-1348.

2. Balaban RB, Williams MV. Improving care transitions: hospitalists partnering with primary care. J Hosp Med. 2010;5(7):375-377. 